\title{
Retraction
}

\section{Retracted: Next Generation Sequencing: Potential and Application in Drug Discovery}

\author{
The Scientific World Journal
}

Received 23 July 2020; Accepted 23 July 2020; Published 25 August 2020

Copyright (C) 2020 The Scientific World Journal. This is an open access article distributed under the Creative Commons Attribution License, which permits unrestricted use, distribution, and reproduction in any medium, provided the original work is properly cited.

The Scientific World Journal has retracted the article titled "Next Generation Sequencing: Potential and Application in Drug Discovery" [1]. The article was found to contain a substantial amount of material from the following published article, cited as reference [15]:

"Recent Patents and Advances in the Next-Generation Sequencing Technologies," by Biaoyang Lin, Jun Wang, and Yin Cheng published in Recent Patents on Biomedical Engineering, 2008, DOI: 10.2174/1874764710801010060 (http://www.eurekaselect.com/95056/article/recent-patentsand-advances-next-generation-sequencing-technologies)

[2].

\section{References}

[1] N. K. Yadav, P. Shukla, A. Omer, S. Pareek, and R. K. Singh, "Next generation sequencing: potential and application in drug discovery," The Scientific World Journal, vol. 2014, Article ID 802437, 7 pages, 2014.

[2] B. Lin, J. Wang, and Y. Cheng, "Recent patents and advances in the next- generation sequencing technologies," Recent Patents on Biomedical Engineering, vol. 1, no. 1, pp. 60-67, 2008. 


\title{
Next Generation Sequencing: Potential and Application in Drug
} Discovery

\author{
Navneet Kumar Yadav, ${ }^{1}$ Pooja Shukla, ${ }^{1,2}$ Ankur Omer, ${ }^{1,2}$ Shruti Pareek, ${ }^{3}$ and R. K. Singh ${ }^{1,2}$ \\ ${ }^{1}$ Hematological Facility, Division of Toxicology, CSIR-Central Drug Research Institute, BS-10/1, Sector 10, Jankipuram Extension, \\ Sitapur Road, P.O. Box 173, Lucknow 226031, India \\ ${ }^{2}$ Academy of Scientific and Innovative Research, New Delhi 110 001, India \\ ${ }^{3}$ Jaipur National University, Jaipur, Rajasthan 302017, India \\ Correspondence should be addressed to R. K. Singh; rktoxi@gmail.com \\ Received 12 August 2013; Accepted 18 October 2013; Published 5 February 2014
}

Academic Editors: Z. P. Çakar and S. Rodríguez-Couto

Copyright (C) 2014 Navneet Kumar Yadav et al. This is an open access article distributed under the Creative Commons Attribution License, which permits unrestricted use, distribution, and reproduction in any medium, provided the original work is properly cited.

The world has now entered into a new era of genomics because of the continued advancements in the next generation high throughput sequencing technologies, which includes sequencing by synthesis-fluorescent in situ sequencing (FISSEQ), pyrosequencing, sequencing by ligation using polony amplification, supported oligonucleotide detection (SOLiD), sequencing by hybridization along with sequencing by ligation, and nanopore technology. Great impacts of these methods can be seen for solving the genome related problems of plant and animal kingdom that will open the door of a new era of genomics. This may ultimately overcome the Sanger sequencing that ruled for 30 years. NGS is expected to advance and make the drug discovery process more rapid.

\section{Introduction}

In 1977 Fred Sanger et al. published two methodology based papers on the rapid determination of DNA sequences that helped to transform biology and provided a new tool for deciphering complete genes and later the entire genome $[1,2]$. The methods dramatically improved existing DNA sequencing techniques developed by Maxam and Gilbert published in the same year and Sanger and Coulson's own "plus and minus" method published 2 years earlier $[3,4]$. The advantage of dealing with less toxic chemicals and radioisotopes made "Sanger sequencing" the only DNA sequencing method used for the next 30 years. The gel based sequencing technology has undergone dramatic improvement in throughput from one parallelization, automation, and refinement of sequencing methods and chemistry. Recent advances in microfibration have resulted in further improvements of Sanger sequencing by multiplexing and miniaturization. These advances have been reviewed by Metzker [5]. Despite many improvements, the gel based Sanger sequencing technology still faces drawbacks in the name of cost and low throughput. For achieving high throughput, many commercial companies and scientific labs have come up with different ways of high throughput sequencing with a reasonable cost.

The technologies named together as next generation sequencing technologies include sequencing by synthesis developed by 454 Life-Science [6]; sequencing by ligation; sequencing by hybridization; single molecule DNA sequencing; nanopore sequencing; and multiplex polony sequencing of George Church's lab [7]. Next generation sequencing has revolutionary impacts on genetic applications like metagenomics, comparative genomics, high throughput polymorphism detection, analysis of small RNAs, mutation screening, transcriptome profiling, methylation profiling, and chromatin remodeling. The major measurements for the success of the next generation technology are sequence (read length), sequence quality, high throughput, and low cost. 
1.1. Sequencing by Synthesis (SBS). SBS using fluorophorelabeled, reversible-terminator nucleotides is the most common platform of sequencing by synthesis. It is sometimes named "fluorescent in situ sequencing" (FISSEQ). The pyrosequencing technology is another SBS technology developed by Ronaghi et al., at Stanford University [8, 9]. It is based on the detection of pyrophosphate (PPi) released during DNA synthesis when inorganic PPi is released after nucleotide incorporation by DNA polymerase. The released $\mathrm{PPi}$ is then converted to ATP by ATP sulfurylase. A luciferase reporter enzyme uses the ATP to generate light, which is then detected by a charged couple device (CCD) camera. Pyrosequencing has evolved into an ultrahigh throughput sequencing technology with the combination of several technologies such as template carrying microbeads deposited in microfabricated picoliter-sized reaction wells connecting to optical fibers [10]. 454 Life Sciences/Roche Diagnostic has Genome Sequencer 20 System and Genome Sequencer FLX System, two high throughput commercial sequencing platforms, and DNA helices are fractionated into 300-500 bp fragments and linkers are added to their $3^{\prime}$ and $5^{\prime}$ ends. Single stranded DNA is isolated and captured on beads. The beads with DNAs are then emulsified in a "water-in-oil" mixture with amplification reagents to create micro reactors for Emulsion PCR (emPCR). Finally beads with amplified DNAs are loaded onto a picotitre plate for sequencing. The procedure is given in Figure 1.

1.2. Polony Sequencing. George Church's Laboratory at Harvard University has developed a high throughput technology based on polony amplification and FISSEQ [11]. Polony amplification is a method to amplify DNA in situ on a thin polyacrylamide film [12]. The DNA movement is limited in the polyacrylamide gel, so the amplified DNAs are localized in the gel and form the so-called "polonies," that is, polymerase colonies (Figure 1). Up to 5 million polonies (i.e., 5 million PCRs) can be formed on a single glass microscope slide. This sequencing technology has been tested in a bacterial genome and the sequence read length was found to be about 13 bases per colony [13]. Kim et al. developed a technology named Polony Multiplex Analysis of Gene Expression (PMAGE), which combines polony amplification and a sequence-by-ligation method, to sequence 14-base tags [14]. Up to 5 million polonies can be sequenced in parallel.

1.3. Single Molecule DNA Sequencing. Most current sequencing technologies are based on sequencing many identical copies of DNA molecules (often amplified). However, there are certain problems associated with sequencing amplified multiple copies of identical DNAs such as achieving the synchronous priming of each copy of the multiple DNA by the sequencing primers [15]. One way to solve such problems is to perform sequencing by synthesis method [16]. DNA can be attached to solid support to form single molecule arrays, and the single DNA molecule is then sequenced directly. Buzby at Helicos Bio-Science Corp. also invented a method of stabilizing a nucleic acid duplex on a surface for single molecule sequencing [17]. Applera Corp. invented fluorescent intercalators that are employed as a donor in fluorescence resonance energy transfer (FRET) for use in single molecule sequencing reactions [18].

1.4. Nanopore Sequencing. Nanopores are nanometer-scale pores and are regarded as one of the most promising technologies in achieving true real time, ultrafast, true single molecule DNA sequencing [19]. Nanopore has been used for the detection, counting, and characterization of single molecules by observing the changes in ionic current in nanopores when molecules traverse through a nanopore $[20,21]$. The fabrication of nanopores such as the alphahemolysin pore and synthetic nanopore has been reviewed recently [22]. Recent progress has made a big step toward ultrafast sequencing using nanopore technologies. Lagerqvist et al. proposed a novel idea to measure the electric current perpendicular to the DNA backbone [23]. Zhao et al. reported that a single nucleotide polymorphism can be detected by a change in the threshold voltage of a nanopore [24].

1.5. Sequencing by Hybridization ( $S B H)$. SBH was given in the late $80 \mathrm{~s}$ [25]. It is a method of reconstructing a DNA sequence based on its k-mer content. All possible k-nucleotides oligomers ( $\mathrm{k}$-mers) are hybridized to identify overlapping $\mathrm{k}$-mers in an unknown DNA sample. These overlapping kmers are subsequently aligned by algorithms to produce the DNA sequence. Drmanac et al. reviewed the technology of sequencing by hybridization [26]. In spite of having some problems, using SBH with predefined probe sets derived from a known sequence has been used to resequence a specific region of genomic DNA or cDNA for the identification of small deletions, insertions, polymorphisms, and mutations [27].

1.6. Sequencing by Ligation. The first high throughput sequencing by ligation was probably realized by Lynx Therapeutics Inc. in their early technology of Massively Parallel Signature Sequencing [28]. Church's laboratory at Harvard Medical School advanced the sequencing by ligation method for ultra fast and high throughput sequencing $[13,14]$. They converted an epifluorescence for rapid DNA sequencing. DNA molecules were amplified in parallel onto micro beads by emulsion polymerase chain reaction (Figure 1). Millions of beads were immobilized in a polyacrylamide gel and sequenced using sequencing by ligation method. Applied Biosystem Inc. acquired Agencourt Personal Genomics who developed the Supported Oligo Ligation Detection system (SOLiD) for high throughput DNA sequencing.

1.7. Bioinformatics Tools for Analyzing NGS Data. Sequence reads generated from NGS technologies are shorter than traditional Sanger sequences, which makes assembly and analysis of NGS data challenging. Some important bioinformatics tools are available which is helpful in analyzing NGS data (Table 1).

1.8. Applications of NGS Techniques. NGS technologies have already been used for various applications, ranging from 


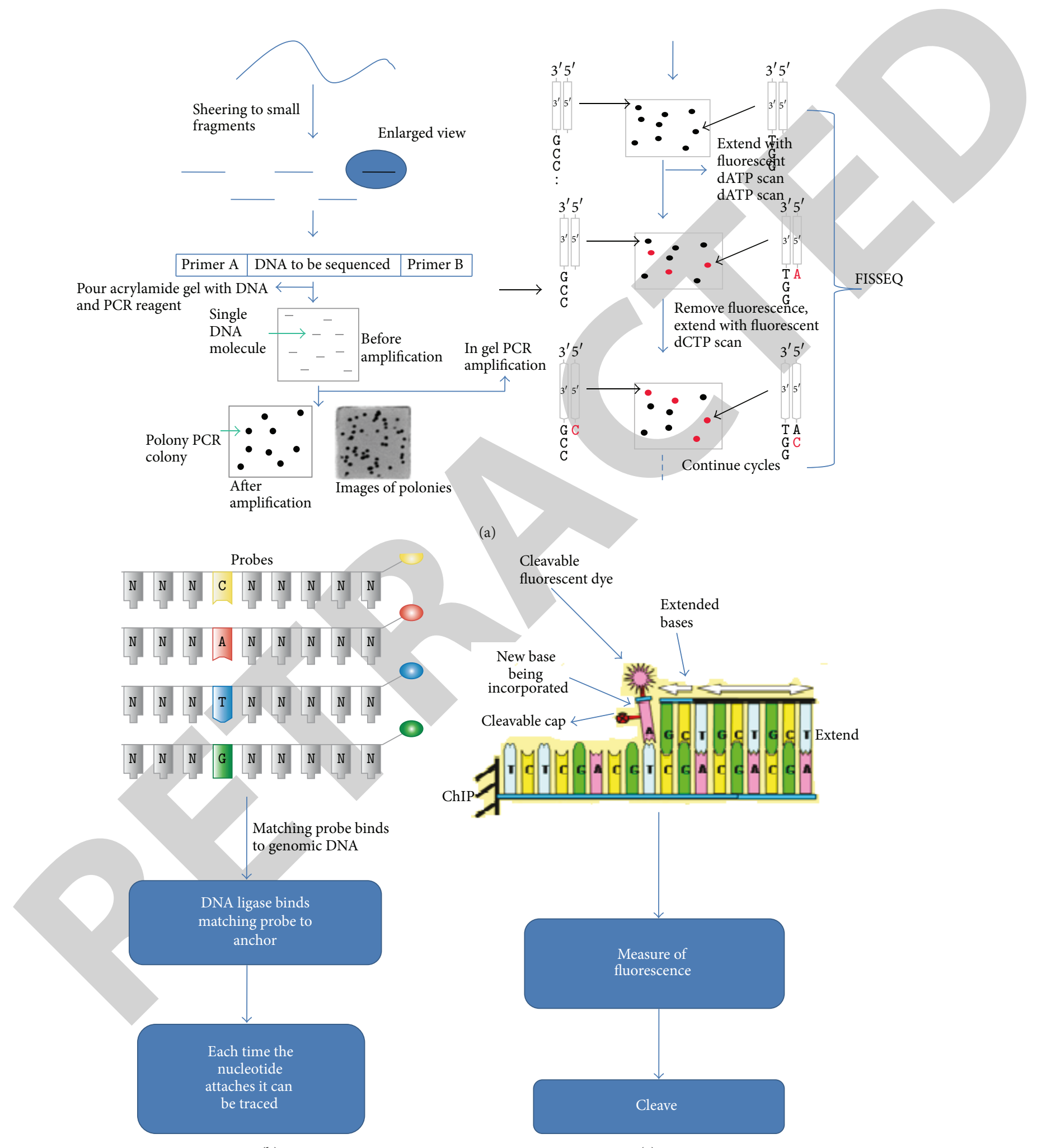

(b)

(c)

FIGURE 1: Different techniques of next generation sequencing: (a) polony sequencing; (b) sequencing by Ligation; (c) sequencing by synthesis. 
TABLE 1: Important bioinformatics tools for analysis of NGS data.

Alignment, assembly, and visualization tools:

Velvet (http://www.ebi.ac.uk/ zerbino/velvet/): tool for de novo assembly of short and paired reads.

EULER (http://ngslib.i-med.ac.at/node/64): tool to generate short-read assembly and facilitate assembly of combined reads of NGS and

Sanger sequencing.

GMAP (http://www.gene.com/share/gmap): program to map and align cDNA sequences to genome sequence using minimal time and memory and facilitates batch processing.

Sequence variant discovery tools:

SNPsniffer tool for SNP discovery specifically designed for Roche/454 sequences.

SeqMap (http://www-personal.umich.edu/ jianghui/seqmap/): tool to map short sequences to a reference genome and detect multiple substitutions and indels.

Integrated tools:

NextGENe (http://www.softgenetics.com/NextGENe.html): software to analyze NGS data for de novo assembly, SNP and indel detection, and transcriptome analysis.

SeqMan genome analyzer (http://www.dnastar.com/products/SMGA.php): software with capacity to align NGS and Sanger data and detect SNPs and also facilitates visualization.

CLCbio Genomics Workbench (http://www.clcbio.com): tool for de novo and reference assembly of Sanger and NGS sequence data, SNP detection and browsing.

Source: http://wiki.seqanswers.com.

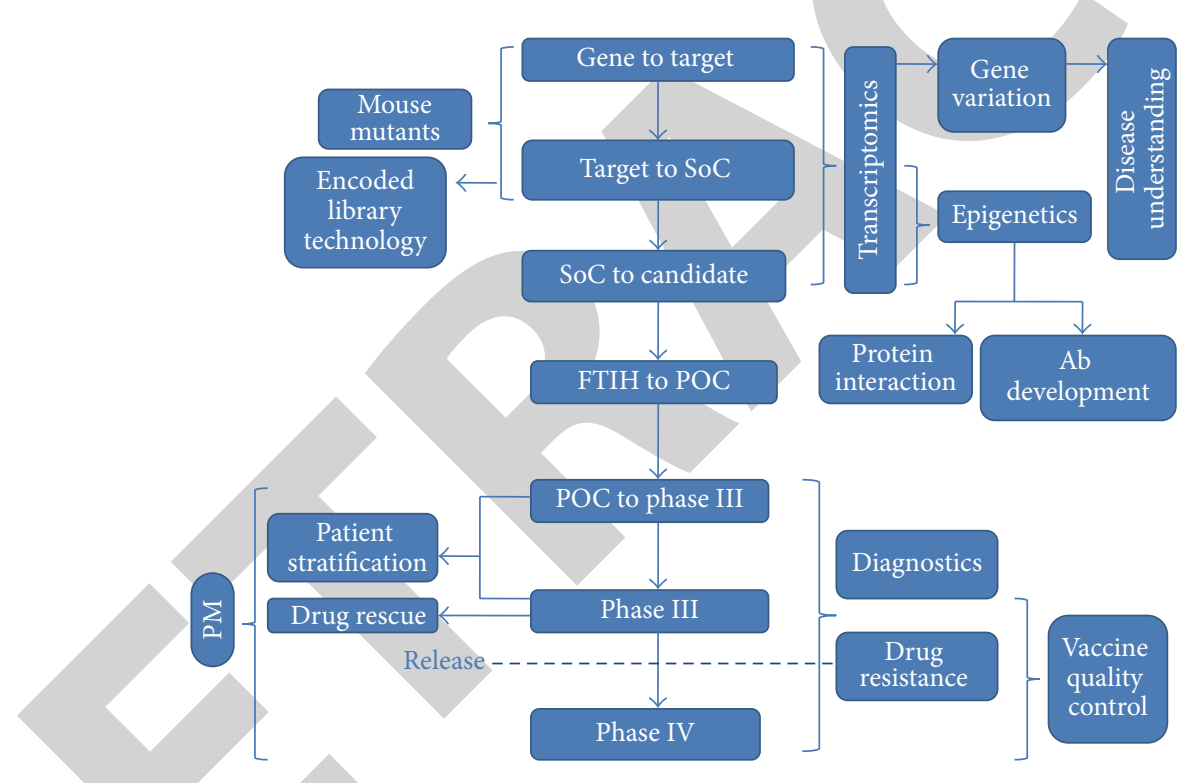

FIGURE 2: Application of Next Generation Sequencing in drug discovery. SoC: standard of care; POC: proof of concept; FTIH: first time in human.

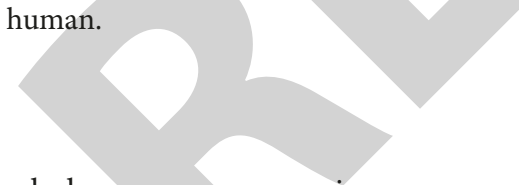

whole genome sequencing, resequencing, single nucleotide polymorphism (SNP), structural variation discovery, mRNA and noncoding RNA profiling, and protein-nucleic acid interaction assays. NGS technologies are becoming a potential tool for gene expression analysis, especially for those species having reference genome sequences already available (Figure 2). An overview of NGS applications that are relevant to drug discovery is discussed here.

1.9. Single Nucleotide Polymorphisms (SNP) Discovery. SNPs are important genomic resources which can be used in a variety of analyses including physical characteristics like height and appearance as well as less obvious traits such as personality, behavior, and disease susceptibility. Sequence data generated for parental genotypes of the mapping population by using NGS technologies can be used for mining the SNPs at large scale [29]. SNPs can also significantly influence responses to pharmacotherapy and predicting whether the drugs will produce adverse reactions or not. The development of new drugs can be made far cheaper and more rapid by selecting participants in drug trials based on their genetically determined response to the drugs [30].

1.10. Messenger RNA and Noncoding RNA Profiling. Apart from SNP discovery, expressed regions of a genome can be detected using NGS technologies. The next generation sequencing platforms are capable of identifying expression levels of nearly all genes, including those rare and species 
specific transcripts. A similar approach can be applied to large genomes. RNA-seq data can be used to characterize exonexon splicing events namely cases of alternative splicing [3133].

Noncoding RNA, like microRNA (miRNA), is a broad class of regulatory RNA molecules.

The NGS technologies are useful for discovery of noncoding RNA for their short lengths [34]. Most studies to date have used 454 technology, because of its early availability to discover new and different noncoding RNA classes in several species like Chlamydomonas, Drosophila, Arabidopsis, and so forth [35-37]. A comprehensive study of miRNA in acute myeloid leukemia was performed using NGS, with novel findings of differentially expressed miRNAs [38]. Transcriptome sequencing has been found to be a powerful tool for detecting novel gene fusions in cancer cell lines and tissues [39].

1.11. De Novo Sequencing and Resequencing. Metagenomics is defined as the application of genomics techniques to directly study the communities of microbial organisms without isolation and cultivation of individual species [40]. It involves the characterization of the genomes in these communities, as well as their mRNA, protein, and metabolic products. The next generation sequencing technologies have enabled to move metagenomics from a single organism type in isolation to the studies of whole communities. NGS enables the researchers to avoid the cloning formation and culture steps which are the major drawbacks of genomics. NGS strategies are straightforward in the following: (1) deep sequencing of DNA fragments is conducted on an uncultured sample and (2) short reads are compared against database of known sequences using bioinformatics tool like MEGAN [41] with or without assembly, and (3) these data are then used to compute and explore their contents to infer relative abundances. Therefore, NGS technologies can be a potent tool for discovery of microorganisms and pathogens.

1.12. Regulatory Protein Binding. At low throughput, chromatin immunoprecipitation (ChIP) has enabled regulatory DNA-protein binding interactions to be elucidated [41]. It is a lengthy process like association of specific antibody to DNA-binding protein, followed by another protein-DNA cross linking agent, so that any protein in close association with DNA becomes linked. Then, the cells are lysed, DNA is fragmented, and the specific antibody is used to precipitate the protein of interest along with any associated DNA fragment. These DNA pieces are subsequently released by reversing the cross linking and identified by southern blotting or qPCR [42]. A probe is used to infer the DNA-binding site sequence of the protein under study. So, NGS helps in identifying the regulatory protein binding site for having shorter reads and specific binding site and it also provides higher resolution [43].

1.13. Exploring Chromatin Packaging. Chromatin packaging denotes the packing of DNA in histones. This packaging determines the transcription of a particular gene. Understanding of this DNA packing, that is, chromatin packaging, is of a great interest. An initial 454-based study of genomic DNA packaging into nucleosomes was described for the $C$. elegans, by sequencing the DNA isolated from nucleosome cores after micrococcal nuclease digestion and mapping them to the reference genome sequence [44]. Mikkelsen et al. used the Illumina platform to demonstrate the connection between chromatin packaging and gene expression in several different cell types. Mikkelsen et al. found that changes in chromatin state at specific promoters reflect changes in gene expression for the genes they control. A better understanding of the chromatin packaging will provide new strategies for the development of novel drugs [45].

NGS can also have a central role in the discovery of new genomic biomarkers, detection of mutations, personalized medicine and pharmacogenetics, target identification and validation, clinical diagnostics, vaccine development, investigating drug resistance and many others.

\section{Conclusion}

Because of their cost-effectiveness in comparison to the Sanger sequencing method and with a wide range of uses NGS approaches have emerged as the dominant genomics technology. Perhaps most significantly, these new sequencers have provided genome-scale sequencing capacity to individual laboratories in addition to larger genome centers. Compared to Sanger sequencing, advantages of the next generation technologies mentioned thus far, including 454/Roche Illumina/Solexa and ABI/SOLiD, alleviate the need for in vivo cloning by clonal amplification of spatially separated single molecules using either emulsion PCR (454/Roche and $\mathrm{ABI} / \mathrm{SOLiD}$ ) or bridge amplification on solid surface (Illumina/Solexa). We are at the verge of a new genomic revolution with recent advances in next generation sequencing technologies. We will see enormous impacts of these next generation sequencing methods in dealing with complex biological problems, for example, the identification of all sequence changes in drug resistant HIV and drug resistant tuberculosis bacteria (TB), in the delineation of sequence changes for individual cells during cancer initiation and progression, and in global transcription factor mapping ChIP to sequencing [46]. In addition, NGS technology will create a tremendous impact in medical science. It will make routine sequencing of a person's genome sequence of an individual human and the initiation of the personal Human Genome Project [47].

\section{Abbreviations}

SBS: $\quad$ Sequencing by synthesis

FISSEQ: Fluorescent in situ sequencing

CCD: Charged couple device

emPCR: Emulsion PCR

PMAGE: Polony multiplex analysis of gene expression

FRET: Fluorescence resonance energy transfer 
SBH: Sequencing by hybridization

k-mers: k-nucleotides oligomers

SOLiD: Supported oligo ligation detection

SNP: Single nucleotide polymorphism

ChIP: Chromatin immunoprecipitation.

\section{Conflict of Interests}

The authors declare that there is no conflict of interests regarding the publication of this paper.

\section{Acknowledgment}

Navneet Kumar Yadav, Ankur Omer and Pooja Shukla acknowledge financial support from UGC and DST, New Delhi. This is CDRI paper no. 74/2012/RKS.

\section{References}

[1] F. Sanger, G. M. Air, B. G. Barrell et al., "Nucleotide sequence of bacteriophage $\varphi$ X174 DNA," Nature, vol. 265, no. 5596, pp. 687-695, 1977.

[2] F. Sanger, S. Nicklen, and A. R. Coulson, "DNA sequencing with chain-terminating inhibitors," Proceedings of the National Academy of Sciences of the United States of America, vol. 74, no. 12, pp. 5463-5467, 1977.

[3] A. M. Maxam and W. A. Gilbert, "New method for sequencing DNA," Proceedings of the National Academy of Sciences of the United States of America, vol. 74, no. 2, pp. 560-564, 1977.

[4] F. Sanger and A. R. Coulson, "Nucleotide and amino acid sequences of Gene G of $\varphi$ X174," Journal of Molecular Biology, vol. 94, pp. 441-448, 1975.

[5] M. L. Metzker, "Emerging technologies in DNA sequencing," Genome Research, vol. 15, no. 12, pp. 1767-1776, 2005.

[6] M. Margulies, M. Egholm, E. William et al., "Genome sequencing in microfabricated high-density picolitre reactors," Nature, vol. 437, pp. 376-380, 2005.

[7] J. J. Shendure, G. J. Porreca, N. B. Reppas et al., "Molecular biology: accurate multiplex polony sequencing of an evolved bacterial genome," Science, vol. 309, no. 5741, pp. 1728-1732, 2005.

[8] M. Ronaghi, S. Karamohamed, B. Pettersson, M. Uhlén, and P. Nyrén, "Real-time DNA sequencing using detection of pyrophosphate release," Analytical Biochemistry, vol. 242, no. 1, pp. 84-89, 1996.

[9] M. Ronaghi, M. Uhlén, and P. Nyrén, "A sequencing method based on real-time pyrophosphate," Science, vol. 281, no. 5375, pp. 363-365, 1998.

[10] J. Shendure, R. D. Mitra, C. Varma, and G. M. Church, "Advanced sequencing technologies: methods and goals," Nature Reviews Genetics, vol. 5, no. 5, pp. 335-344, 2004.

[11] J. Shendure and H. Ji, "Next-generation DNA sequencing," Nature Biotechnology, vol. 26, no. 10, pp. 1135-1145, 2008.

[12] R. D. Mitra and G. M. Church, "In situ localized amplification and contact replication of many individual DNA molecules," Nucleic Acids Research, vol. 27, no. 24, article e34, 1999.

[13] S. Myllykangas, J. Buenrostro, and H. P. Ji, "Overview of sequencing technology platforms," Bioinformatics For High Throughput Sequencing, pp. 11-25, 2012.
[14] J. B. Kim, G. J. Porreca, L. Song et al., "Polony multiplex analysis of gene expression (PMAGE) in mouse hypertrophic cardiomyopathy," Science, vol. 316, no. 5830, pp. 1481-1484, 2007.

[15] B. Lin, J. Wang, and Y. Cheng, "Recent patents and advances in the next-generation sequencing technologies," Recent Patents on Biomedical Engineering, vol. 1, pp. 60-67, 2008.

[16] S. N. Lapidus, P. R. Buzby, and T. Harris, US20077169560, 2007.

[17] P. R. Buzby, US20077220549, 2007.

[18] H. Sun, US20070202521A1, 2007.

[19] M. Rhee and M. A. Burns, "Nanopore sequencing technology: research trends and applications," Trends in Biotechnology, vol. 24, no. 12, pp. 580-586, 2006.

[20] A. Meller and D. Branton, "Single molecule measurements of DNA transport through a nanopore," Electrophoresis, vol. 23, pp. 2583-2591, 2002.

[21] A. Meller, L. Nivon, E. Brandin, J. Golovchenko, and D. Branton, "Rapid nanopore discrimination between single polynucleotide molecules," Proceedings of the National Academy of Sciences of the United States of America, vol. 97, no. 3, pp. 1079-1084, 2000.

[22] M. Rhee and M. A. Burns, "Nanopore sequencing technology: nanopore preparations," Trends in Biotechnology, vol. 25, no. 4, pp. 174-181, 2007.

[23] J. Lagerqvist, M. Zwolak, and M. di Ventra, "Fast DNA sequencing via transverse electronic transport," Nano Letters, vol. 6, no. 4, pp. 779-782, 2006.

[24] Q. Zhao, G. Sigalov, V. Dimitrov et al., "Detecting SNPs using a synthetic nanopore," Nano Letters, vol. 7, no. 6, pp. 1680-1685, 2007.

[25] D. Radoje, L. Ivan, B. Ivan, and C. Radomir, "Sequencing of megabase plus DNA by hybridization: theory of the method," Genomics, vol. 4, pp. 114-128, 1989.

[26] R. Drmanac, S. Drmanac, G. Chui et al., "Sequencing by hybridization (SBH): advantages, achievements, and opportunities," Advances in Biochemical Engineering/Biotechnology, vol. 77, pp. 75-101, 2002.

[27] A. Schirinzi, S. Drmanac, B. Dallapiccola et al., "Combinatorial sequencing-by-hybridization: analysis of the NF1 gene," Genetic Testing, vol. 10, no. 1, pp. 8-17, 2006.

[28] S. Brenner, S. R. Williams, E. H. Vermaas et al., "In vitro cloning of complex mixtures of DNA on microbeads: physical separation of differentially expressed cDNAs," Proceedings of the National Academy of Sciences of the United States of America, vol. 97, no. 4, pp. 1665-1670, 2000.

[29] V. Thakur and R. Varshney, "Challenges and strategies for next generation sequencing (NGS) data AR," Journal of Computer Science and Systems Biology, vol. 3, pp. 40-42, 2010.

[30] J. Voisey and C. P. Morris, "SNP technologies for drug discovery: a current review," Current Drug Discovery Technologies, vol. 5, no. 3, pp. 230-235, 2008.

[31] M. Samuel, B. T. Wilhelm, and J. Bähler, "Next-generation sequencing: applications beyond Genomes," Biochemical Society Transactions, vol. 36, pp. 1091-1096, 2008.

[32] U. Nagalakshmi, Z. Wang, K. Waern et al., "The transcriptional landscape of the yeast genome defined by RNA sequencing," Science, vol. 320, no. 5881, pp. 1344-1349, 2008.

[33] M. Sultan, M. H. Schulz, H. Richard et al., "A global view of gene activity and alternative splicing by deep sequencing of the human transcriptome," Science, vol. 321, no. 5891, pp. 956-960, 2008. 
[34] M. Kiriakidou, P. T. Nelson, A. Kouranov et al., "A combined computational-experimental approach predicts human microRNA targets," Genes and Development, vol. 18, no. 10, pp. 1165-1178, 2004.

[35] J. Brennecke, A. A. Aravin, A. Stark et al., "Discrete small RNAgenerating loci as master regulators of transposon activity in Drosophila," Cell, vol. 128, no. 6, pp. 1089-1103, 2007.

[36] S. Alexander, K. Pouya, P. Leopold et al., "Systematic discovery and characterization of fly microRNAs using 12 Drosophila genomes," Genome Research, vol. 17, no. 12, pp. 1865-1879, 2007.

[37] K. D. Kasschau, N. Fahlgren, E. J. Chapman et al., "Genomewide profiling and analysis of Arabidopsis siRNAs," PLoS Biology, vol. 5, no. 3, article 57, 2007.

[38] G. Ramsingh, D. C. Koboldt, M. Trissal et al., "Complete characterization of the microRNAome in a patient with acute myeloid leukemia," Blood, vol. 116, no. 24, pp. 5316-5326, 2010.

[39] C. A. Maher, C. Kumar-Sinha, X. Cao et al., "Transcriptome sequencing to detect gene fusions in cancer," Nature, vol. 458, no. 7234, pp. 97-101, 2009.

[40] K. Chen and L. Pachter, "Bioinformatics for whole-genome shotgun sequencing of microbial communities," PLoS Computational Biology, vol. 1, no. 2, article e24, 2005.

[41] D. H. Huson, A. F. Auch, J. Qi, and S. C. Schuster, "MEGAN analysis of metagenomic data," Genome Research, vol. 17, no. 3, pp. 377-386, 2007.

[42] M. J. Solomon, P. L. Larsen, and A. Varshavsky, "Mapping protein-DNA interactions in vivo with formaldehyde: evidence that histone $\mathrm{H} 4$ is retained on a highly transcribed gene," Cell, vol. 53, no. 6, pp. 937-947, 1988.

[43] E. R. Mardis, "The impact of next-generation sequencing technology on genetics," Trends in Genetics, vol. 24, no. 3, pp. 133-141, 2008.

[44] S. M. Johnson, F. J. Tan, H. L. McCullough, D. P. Riordan, and A. Z. Fire, "Flexibility and constraint in the nucleosome core landscape of Caenorhabditis elegans chromatin," Genome Research, vol. 16, no. 12, pp. 1505-1516, 2006.

[45] T. S. Mikkelsen, M. Ku, D. B. Jaffe et al., "Genome-wide maps of chromatin state in pluripotent and lineage-committed cells," Nature, vol. 448, no. 7153, pp. 553-560, 2007.

[46] D. S. Johnson, A. Mortazavi, R. M. Myers, and B. Wold, "Genome-wide mapping of in vivo protein-DNA interactions," Science, vol. 316, no. 5830, pp. 1497-1502, 2007.

[47] S. Levy, G. Sutton, P. C. Ng et al., "The diploid genome sequence of an individual human," PLoS Biology, vol. 5, no. 10, article e254, 2007. 